\title{
Current and future perspectives for dentin-pulp tissue engineering - an update
}

SADJ April 2019, Vol. 74 No. 3 p110 - p114

\section{S Srivastava}

\section{SUMMARY}

The isolation of dental pulp stem cells in 2000 set the scene enabling tissue engineering to generate dental pulp, leading to the possibility of further root development and the reinforcement of dentinal walls by deposition of hard tissues. These developments would be of clinical interest for dentists in endodontics, in which exciting areas of endodontic research are emerging. In roots having thin dentinal walls, there is a risk of fracture when mechanical preparation of the root canal is undertaken. The innovative option of regenerative endodontic procedures could prove to be an effective approach in these cases. This review is an attempt to update on the current trends.

\section{INTRODUCTION}

Regenerative endodontics provides the hope of converting the non-vital tooth into the vital state once again, focusing on substituting the traumatized and pathological pulp with functional pulp tissue. The technique has been defined as "a biologically-based procedure designed to physiologically replace damaged tooth structures, including dentin and root structures, as well as cells of the pulp-dentin complex". ${ }^{1}$

The foundation for regenerative endodontic procedures (REPs) was established by Nygaard-Ostby who observed that the formation of a blood clot was not only desirable, but also sought after as a means of ensuring adequate healing. ${ }^{2}$ Despite some shortcomings, this pioneer study laid the foundation for subsequent investigations in the field of regenerative endodontics. In 2004, Banchs and Trope $^{3}$ published a case report describing a new treatment procedure in the management of the open apex, called "revascularization". Unlike traditional apexification or the use of apical barriers, revascularization procedures allow for an increase in both the length of the root and the thickness of the root wall. Hargreaves et al. recommended the interplay of stem cells, bioactive molecules and bioactive scaffolds, forming a triad during REP therapies, which aims to reestablish the microenvironments suitable for regeneration of desired tissue. ${ }^{4}$

\section{Author affiliations:}

Swati Srivastava: BDS, MDS (Endodontics), Assistant Professor, Department of Conservative Dental Sciences, College of Dentistry, Qassim University, Buraydah, Kingdom of Saudi Arabia.

Corresponding author: Swati Srivastava

Department of Conservative Dental Sciences, College of Dentistry,

Qassim University, Buraydah, Kingdom of Saudi Arabia.

Email: swatisrivastava412@gmail.com

\author{
ACRONYMS \\ BMP: Bone Morphogenic Protein \\ DPSC: Dental Pulp Stem Cells \\ LIPUS: Low-Intensity Pulsed Ultrasound \\ MSC: Mesenchymal Stem Cells \\ NETs: Neutrophil Extracellular Traps \\ PRF: Platelet-Rich Fibrin \\ PRP: Platelet-Rich Plasma \\ REPs: Regenerative Endodontic Procedures \\ SCAP: Stem Cells of Apical Papilla \\ VEGF: Vascular Endothelial Growth Factor
}

A dental stem cell is a self-renewable cell type in the tooth, involved in developing, or in the maintenance of adult dental tissues. Their growth and differentiation are dependent on the release by the dentine niche of growth factors or bioactive molecules. The dentine niche describes an environment conducive to tissue regeneration. The success of vital pulp therapies like pulp capping and pulpotomy are based on the ability of surviving stem cells of the remaining vital dental pulp to help in repair. A dental niche having even small fragments of residual vital pulp tissue will increase the success of regeneration of the damaged tissue. ${ }^{10}$

Bioactive molecules are signaling molecules or chemical cues that control a variety of cellular responses through specific binding of transmembrane or intracellular receptors in a target cell. ${ }^{5}$ The bone morphogenic protein (BMP) superfamily of proteins such as BMP-2, BMP-4, BMP-7, and transforming growth factor beta 1(TGF- $\beta 1$ ) are found to modulate cellular functions. ${ }^{6}$ There exists a rich cocktail of bioactive molecules in dentin and pulp capable of giving the signaling events involved in dentin and pulp regeneration. ${ }^{7}$

Bioactive scaffolds should facilitate cellular infiltration, vascularization, cell differentiation, and also be biodegradable, allowing eventual replacement by the appropriate tissues. The variety of biomaterials include:

1. Tissue extracts such as blood clot, platelet- rich plasma (PRP), platelet- rich fibrin (PRF), pure plateletrich fibrin, and leukocyte platelet-rich fibrin;

2. Ceramics such as calcium hydroxyapatite, tricalcium phosphate, and mineral trioxide aggregate;

3. Synthetic polymers such as polylactic and polylactic-co-glycolic acids;

4. Biopolymers such as collagen, hyaluronan and chitosan; and self-assembling peptide hydrogel. 
Most of the biomaterials that have been proposed lack approval from the Food and Drug Administration (FDA) which makes them not suitable for clinical use. ${ }^{8}$ Chrepa et al. demonstrated in vitro an FDA-approved Hyaluronic acid hydrogel -Restylane, an injectable scaffold which promoted the survival, mineralization, and differentiation of stem cells of the apical papilla (SCAP) into an odontoblastic phenotype. As this product, Restylane, is available in injectable form, it may represent a suitable scaffold for immediate clinical use in endodontic regenerative procedures. ${ }^{9}$

\section{A new bioengineering dental triad}

The traditional triad of dental stem cells, bioactive signal molecules and bioactive scaffolds as mentioned above fails to generate healthy functional tissue. This is because the regenerated tissue does not completely match the normal healthy functional tissue. These unsatisfactory results suggest that some important factors are still missing in current REPs. Hence, a new dental bioengineering approach has been suggested (Figure 1). The concept involves providing microenvironments favourable to signal molecules and to stem cells.

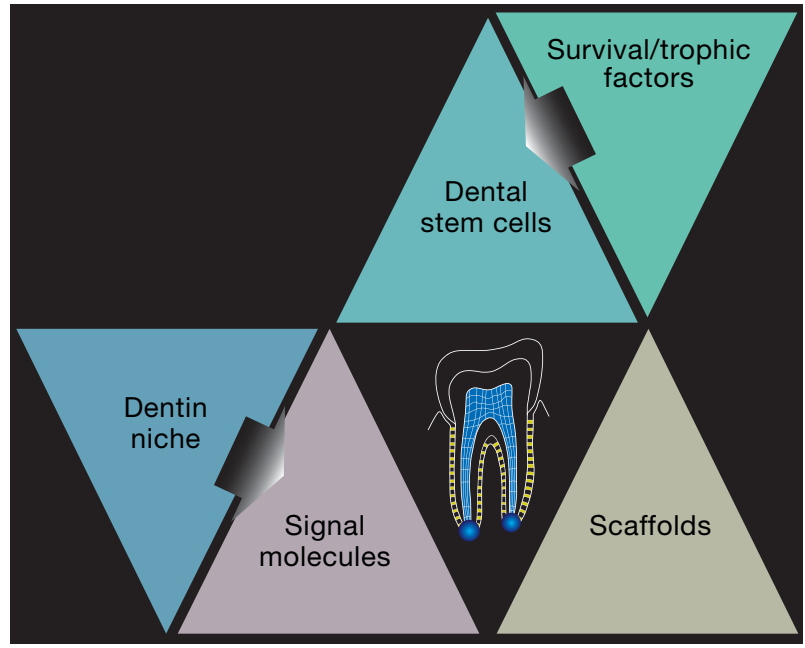

Figure 1. A new dental bioengineering approach with dentin niche and trophic factors.

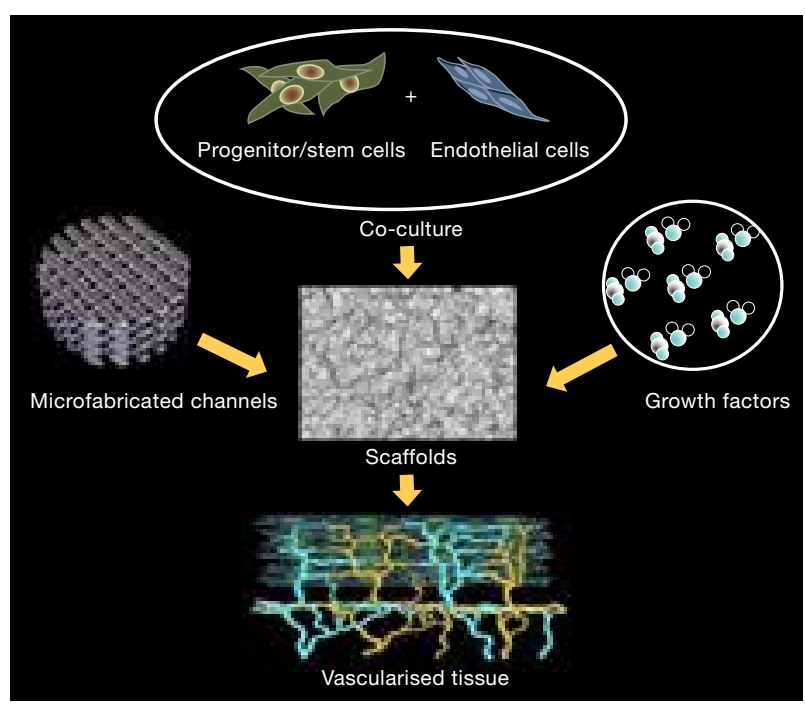

Figure 2. Potential approaches of enhancing vasculature in tissue engineering.
Trophic factors/Survival signals

The dentin matrix contains a reservoir of growth factors and other bioactive molecules sequestered or "fossilized" within the mineralized matrix. These growth factors regulate cell proliferation and differentiation during tooth development and become entrapped in the dentin matrix where they remain functional during life. ${ }^{11}$

Dentin-entrapped factors are potentially controlled by trophic factors regulating "positional memory" during development. Their exact concentrations could be highly heterogeneous. General REP treatments may be benefited by the release of these entrapped factors from the dentin.

Current developments and challenges in Regenerative Endodontics

\section{Vasculature engineering}

Angiogenesis is important not only for nutrient supply during regeneration of the tissue but also, potentially, for stem cell recruitment. During the last decade, there have been major advances in the understanding of the process of neo-angiogenesis which can be categorized into three main approaches. ${ }^{12}$

\section{Incorporation of growth factors}

Angiogenic factor-incorporated scaffolds incoporating sustained release Vascular Endothelial Growth Factor (VEGF) is a signal protein which is a most potent angiogenic and vasculogenic factor, promoting endothelial cell proliferation, migration, and survival. Yadlapati et al. $^{13}$ found that VEGF-loaded biodegradable fibre may be considered a viable option to stimulate angiogenesis and new tissue formation during endodontic regeneration procedures.

\section{Co-culture of progenitor/target cells with endothelial cells}

A number of studies have shown that the co-culture of stem/progenitor cells with endothelial/endothelial progenitor cells significantly enhances angiogenesis, acting synergistically. ${ }^{14,15}$ Findings indicate that the co-culture of dental pulp stem cells (DPSCs) and endothelial cells is a promising source for regenerative endodontics.

\section{Microfabrication of vasculature or de-cellularized matrices}

This approach aims to create a blueprint for microcirculation in a biodegradable scaffold so that endothelial cells attach to microchannels and proliferate, giving rise to a functional vasculature. ${ }^{16,17}$ Although this method has been investigated in relation to engineering complex tissues such as the liver and brain, it is yet to be attempted in dental tissue engineering.

This approach could overcome one of the most common constraints in pulp regeneration strategies, namely the lack of perfusion at the periphery of newly implanted pulp tissue, which hinders full-length pulp regeneration.

\section{Pulp tissue graft}

Minced pulp tissue has been used as a source of mesenchymal stem cells (MSC) for tissue regeneration.

The transplanted tissue yields migrating cells that retain 
the potential for odontogenic and osteogenic differentiation, allowing for de novo pulp-dentin regeneration, and bypassing the need for in vitro culture.

This is the basis of the proposed protocol for the "pulp tissue grafting" approach to regenerate the pulp-dentin complex in teeth that require endodontic intervention, without the need for in vitro culture of pulpal stem cells. ${ }^{18}$

\section{Low-intensity pulsed ultrasound (LIPUS) treatment} MSC from dental tissues may respond to low-intensity pulsed ultrasound treatment (LIPUS), potentially providing a therapeutic approach to promoting dental tissue regeneration. ${ }^{19}$

The mechanism has not yet been fully elucidated but is attributed to its non-thermal biomechanical affects. In particular, through acoustic microstreaming and physical radiation, LIPUS may influence the cell membrane and cytoskeleton to trigger downstream signalling processes. Therefore, this relatively easy and inexpensive application may provide an ideal therapy in the dental clinic for the regeneration of dental tissues..$^{19,20}$

\section{Effect of infection on apical papilla}

The apical papilla undergoes liquefaction necrosis along with the dental pulp because they are interconnected. Recent evidence shows that the apical papilla and its rich resident stem cells are capable of surviving prolonged endodontic infections which may have lead to apical periodontitis. The apical papilla represents a rich source of undifferentiated mesenchymal stem cells in REPs. ${ }^{21}$

The survival of the apical papilla and its stem cell population might explain why MSCs such as SCAP can survive during apical periodontitis.
The biological reason for survival may be explained by the relatively low density of blood vessels in the apical papilla in comparison with the adjacent dental pulp.

\section{Disinfection vs regeneration}

Most REPs include minimal or no mechanical debridement. Hence, there is heavy reliance on the chemical debridement. The irrigants used should be selected on the basis of their bactericidal/bacteriostatic properties and their ability to promote survival and proliferation of stem cells. ${ }^{22}$ Thus, an important concept of regenerative endodontics is to achieve disinfection while creating conditions conducive to stem cell survival, proliferation, and differentiation. It has been demonstrated that $6 \% \mathrm{NaOCl}$ denatures the growth factors embedded in dentin. However, EDTA helps in dissolving these growth factors out of the dentin, thereby increasing their bioavailability. ${ }^{23}$ Importantly, the growth factors embedded in the dentin matrix such as TGF-b and dentin sialoprotein (DSP) are known to be potent stimulators of stem cell proliferation and differentiation. ${ }^{24}$

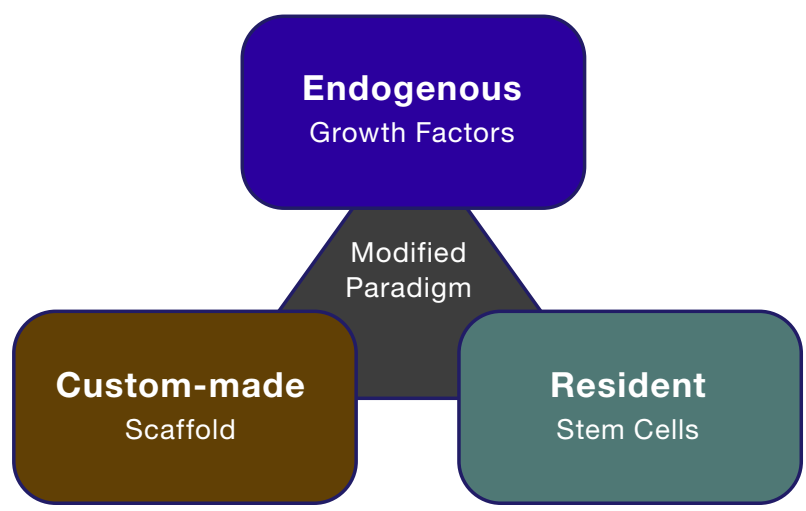

Figure 3. Tissue engineering paradigm modified for cell-homing approaches of pulp regeneration.

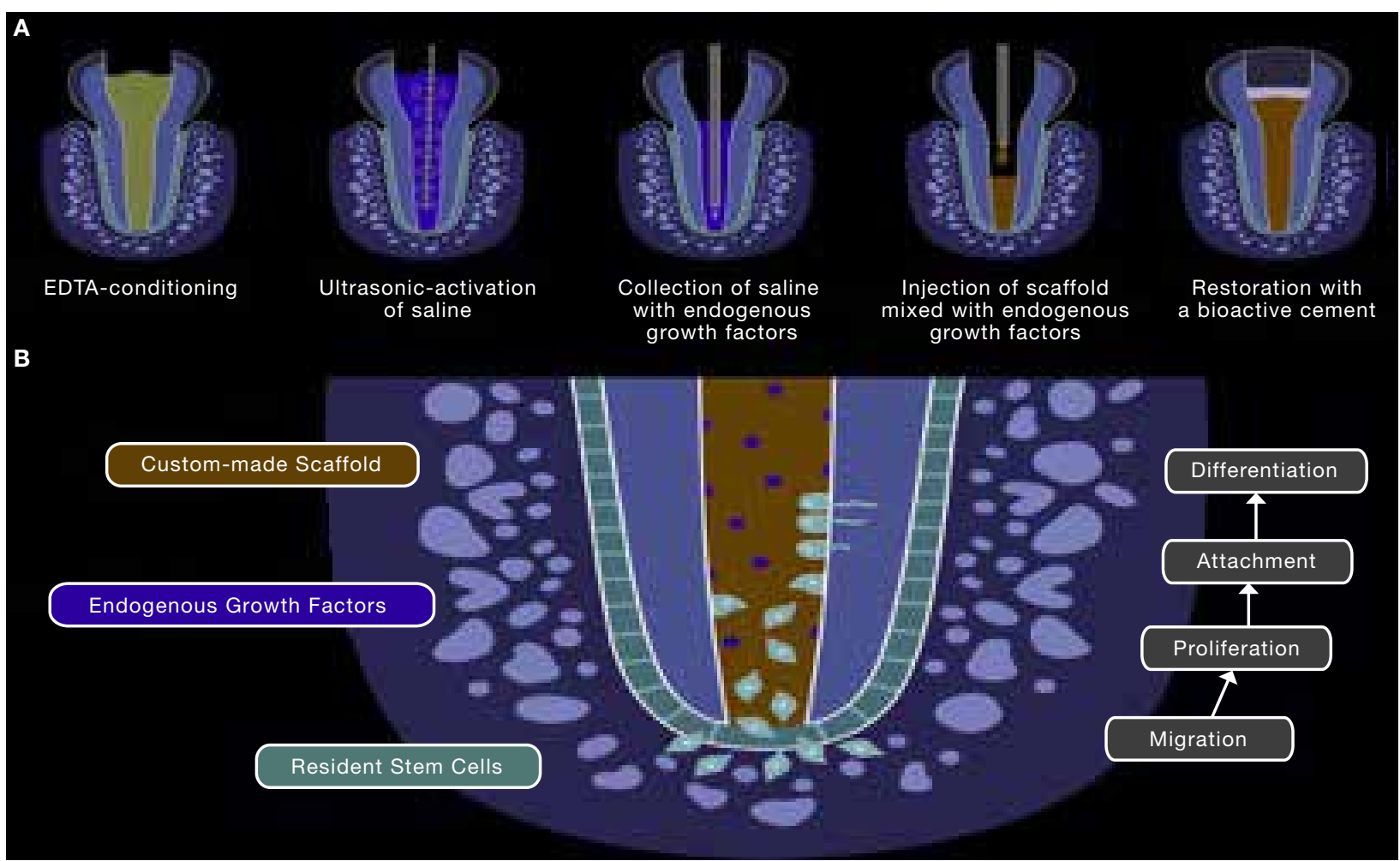

Figure 4. Clinical management of a cell-homing approach to engineer dental pulp. 


\section{Potential role of NETs \\ (Neutrophil Extracellular Traps)}

Recently, a novel bacterial killing mechanism termed neutrophil extracellular traps (NETs) has been described that uses reactive oxygen species signalling and results in cellular DNA extrusions causing microbial entrapment and death. ${ }^{25}$

NETs and their components, such as histones, may provide novel prognostic markers for pulp pathologies. The determination of their levels within the infected pulp could be exploited to target the application of novel disease management strategies. They have a potential role in controlling pulpal infections. How these structures may influence the pulp's vitality and regenerative responses is an area of further research.

\section{Cell-homing approach: regenerative endodontics for adult patients}

Tremendous effort has been made to revitalize disinfected immature permanent teeth in children and adolescents with diagnoses including pulp necrosis or apical periodontitis. The question remains whether regenerative endodontics can be extended to revitalize mature permanent teeth with diagnoses including irreversible pulpitis and/or pulp necrosis in adults.

The concept of cell homing in dental pulp and dentin regeneration was first proposed in $2010 .{ }^{26}$ In tissue regeneration, cell homing includes two separate cellular processes: cell recruitment and differentiation.

Recruitment is directional cell migration to the tissue injury or defects. Differentiation indicates the process of transformation of stem/progenitor cells into increasingly mature and matrix synthesis cells. In dental pulp and dentin regeneration, stem/progenitor cells differentiate into odontoblasts, pulp fibroblasts, and other niche cells. ${ }^{27}$ Cell homing shows a potential for dental pulp regeneration in mature permanent teeth in adults, as shown by He et al. ${ }^{28}$

The development of biologically-based approaches to regenerate or repair dental pulp has become possible because of recent advances in tissue engineering and biomaterials. However, the several severe problems associated with this approach may render it not feasible for a clinical setting, at least in the near future. Galler et al. ${ }^{24}$ suggested a modification of the classical tissue engineering paradigm (Figure 3), where resident cells are attracted by endogenous, dentin derived growth factors and induce further cell proliferation and differentiation. A bioactive scaffold material laden with these growth factors would serve as a template for tissue formation (Figure 4).

\section{What the future holds? Immediate versus delayed} induction:

Currently, there are no reports of randomized clinical trials on immediate induction for regenerative endodontics, despite the need for investigation in this field. Such as have been conducted, which have compared immediate induction versus delayed induction protocols, show clinical success rates of $71 \%$ for delayed induction and $33 \%$ for immediate induction. ${ }^{29}$
The association between success rate and etiology are possible predictor factors. At the time pulp necrosis is diagnosed, the stage of root development might be a relevant factor for periapical and apical healing.

Unfortunately, there is no information on the stage of root development at the time of induction for most studies. Determination of the stage of root formation and etiology are possible critical factors for any therapeutic decision.

\section{DISCUSSION}

The field of regenerative endodontics is evolving.

Present concepts of the regeneration of dental tissues can revolutionize endodontic procedures. However, there is still considerable debate as to what represents clinical success with such procedures. The source and potency of pulpal mesenchymal stem cells are of paramount importance and could be limiting factors in making this technology available for routine endodontic therapies for patients. Key research questions which still need to be answered are:

a). How do we address the challenges involved in the preparation and quality control of clinical grade dental pulp stem cells, which are safe and show efficacy?

b). If replacement teeth were available today from a hypothetical tooth tissue bank would it be a practical option for every restorative circumstance?

c). Are cell homing protocols feasible for clinical success, especially without the introduction of exogenous signalling molecules?

Regenerative procedures could eventually promote tooth longevity in our aging population. Further clinical studies could make significant progress toward achievement of good patient-based, clinician-based and scientist based outcomes.

\section{References}

1. Murray PE, Garcia-Godoy F, Hargreaves KM. Regenerative endodontics: a review of current status and a call for action. J Endod. 2007;33(4):377-90.

2. Östby BN. The role of the blood clot in endodontic therapy: an experimental histologic study. Acta Odontologica Scandinavica. 1961;19(3-4):323-53.

3. Banchs F, Trope M. Revascularization of immature permanent teeth with apical periodontitis: new treatment protocol? J Endod. 2004;30(4):196-200.

4. Hargreaves KM, Diogenes A, Teixeira FB. Treatment options: Biological basis of Regenerative Endodontic Procedures. Journal of Endodontics.39(3):S30-S43.

5. Lee K, Silva EA, Mooney DJ. Growth factor delivery-based tissue engineering: general approaches and a review of recent developments. Journal of the Royal Society, Interface. 2011;8(55):153-70.

6. Nakashima M, Nagasawa H, Yamada Y, Reddi AH. Regulatory role of transforming growth factor-beta, bone morphogenetic protein-2, and protein-4 on gene expression of extracellular matrix proteins and differentiation of dental pulp cells. Developmental Biology. 1994;162(1):18-28.

7. Smith AJ, Duncan HF, Diogenes A, Simon S, Cooper PR. Exploiting the bioactive properties of the dentin-pulp complex in Regenerative Endodontics. J Endod. 2016;42(1):47-56.

8. Galler KM, D'Souza RN, Hartgerink JD, Schmalz G. Scaffolds for dental pulp tissue engineering. Adv Dent Res. 2011;23(3):333-9. 
9. Chrepa V, Austah O, Diogenes A. Evaluation of a commercially available hyaluronic acid hydrogel (Restylane) as injectable scaffold for dental pulp regeneration: an in vitro evaluation. J Endod. 2017;43(2):257-62.

10. Huang GT, Yamaza T, Shea LD, Djouad F, Kuhn NZ, Tuan RS, et al. Stem/progenitor cell-mediated de novo regeneration of dental pulp with newly deposited continuous layer of dentin in an in vivo model. Tissue Engineering Part A. 2010;16(2):605-15.

11. Salehi S, Cooper P, Smith A, Ferracane J. Dentin matrix components extracted with phosphoric acid enhance cell proliferation and mineralization. Dental Materials: official publication of the Academy of Dental Materials. 2016;32(3):334-42.

12. Dissanayaka WL, Zhang $C$. The role of vasculature engineering in dental pulp regeneration. J Endod. 2017;43(9s):S102-s6.

13. Yadlapati M, Biguetti C, Cavalla F, Nieves F, Bessey C, Bohluli P, et al. Characterization of a vascular endothelial growth factorloaded bioresorbable delivery system for pulp regeneration. J Endod. 2017:43(1):77-83.

14. Melero-Martin JM, De Obaldia ME, Kang SY, Khan ZA, Yuan $L$, Oettgen $P$, et al. Engineering robust and functional vascular networks in vivo with human adult and cord blood-derived progenitor cells. Circulation Research. 2008;103(2):194-202.

15. Aguirre A, Planell JA, Engel E. Dynamics of bone marrowderived endothelial progenitor cell/mesenchymal stem cell interaction in co-culture and its implications in angiogenesis. Biochemical and Biophysical Research Communications. 2010;400(2):284-91.

16. Morgan JP, Delnero PF, Zheng Y, Verbridge SS, Chen J, Craven $\mathrm{M}$, et al. Formation of microvascular networks in vitro. Nature Protocols. 2013:8(9):1820-36.

17. Kaully T, Kaufman-Francis K, Lesman A, Levenberg S. Vascularization - the conduit to viable engineered tissues. Tissue Engineering Part B, Reviews. 2009;15(2):159-69.

18. Liang Z, Kawano S, Chen W, Sadrkhani MS, Lee C, Kim E, et al. Minced pulp as source of pulpal mesenchymal stem cells with odontogenic differentiation capacity.

J Endod. 2018;44(1):80-6.
19. Man J, Shelton RM, Cooper PR, Scheven BA. Low-intensity low-frequency ultrasound promotes proliferation and differentiation of odontoblast-like cells. J Endod. 2012;38(5):608-13.

20. Scheven BA, Shelton RM, Cooper PR, Walmsley AD, Smith AJ. Therapeutic ultrasound for dental tissue repair. Medical Hypotheses. 2009;73(4):591-3.

21. Diogenes A, Hargreaves KM. Microbial modulation of stem cells and future directions in Regenerative Endodontics. J Endod. 2017;43(9s):S95-s101.

22. Trevino EG, Patwardhan AN, Henry MA, Perry G, DybdalHargreaves N, Hargreaves KM, et al. Effect of irrigants on the survival of human stem cells of the apical papilla in a platelet-rich plasma scaffold in human root tips. J Endod. 2011;37(8):1109-15.

23. Pang NS, Lee SJ, Kim E, Shin DM, Cho SW, Park W, et al. Effect of EDTA on attachment and differentiation of dental pulp stem cells. J Endod. 2014;40(6):811-7.

24. Galler KM, Eidt A, Schmalz G. Cell-free approaches for dental pulp tissue engineering. J Endod. 2014;40(4 Suppl):S41-5.

25. Cooper PR, Chicca IJ, Holder MJ, Milward MR. Inflammation and regeneration in the dentin-pulp complex: Net gain or net loss? J Endod. 2017;43(9s):S87-s94.

26. Lee CH, Cook JL, Mendelson A, Moioli EK, Yao H, Mao JJ. Regeneration of the articular surface of the rabbit synovial joint by cell homing: a proof of concept study. Lancet (London, England). 2010;376(9739):440-8.

27. Kim JY, Xin X, Moioli EK, Chung J, Lee CH, Chen M, et al. Regeneration of dental-pulp-like tissue by chemotaxis-induced cell homing. Tissue Engineering Part A. 2010;16(10):3023-31.

28. He L, Kim SG, Gong Q, Zhong J, Wang S, Zhou X, et al. Regenerative Endodontics for adult patients. J Endod. 2017;43(9s):S57-s64.

29. Botero TM, Tang X, Gardner R, Hu JCC, Boynton JR, Holland GR. Clinical evidence for regenerative endodontic procedures: immediate versus delayed induction? J Endod. 2017;43(9s):S75-s81. 